\title{
REGIONAL RATES OF SWEAT EVAPORATION DURING LEG AND ARM CYCLING
}

\author{
J. H. AYLING, PhD
}

Lecturer in Exercise Physiology, Department of Physical Education, University of Leeds, Leeds LS2 9JT

\section{ABSTRACT}

The rate of sweat evaporation from the arm, chest, back and thigh, aural temperature, skin temperature (arm, chest, back and thigh), heat production (derived from measurements of respiratory gas exchange) and heart rate were measured in 7 men during 15 minutes of leg or arm cycling at $32 \%$ of predicted maximum oxygen uptake $\left(\mathrm{VO}_{2}\right.$ max). The regional sweat evaporation rates and changes in body temperature were similar during both forms of exercise. The peak rates of sweat evaporation

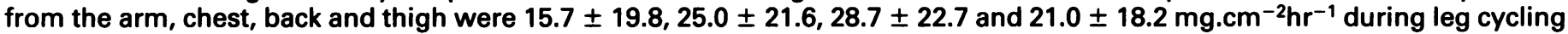

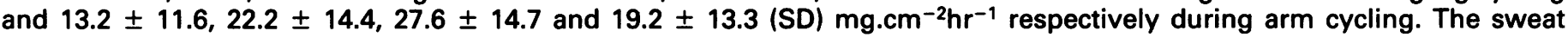
evaporation rates from the different body regions were not significantly different from one another.

Key words: Sweat evaporation, Body temperature, Exercise.

\section{INTRODUCTION}

The initial sweating response to exercise occurs within 1.5 seconds in a warm environment $\left(37.5^{\circ} \mathrm{C}\right)$. The speed of this response suggests that it is not a thermoregulatory one (Beaumont and Bullard, 1963). The fully developed, i.e. steady-state, response is not seen until 30-40 minutes after the start of exercise (Nielsen, 1969). Since the later sweating response is thermoregulatory it is not surprising that a reasonable prediction of an individual's sweat production rate can be made from measurements of deep body and mean skin temperature (Davies, 1979).

However, heat is lost through sweating only when sweat evaporates. In cases where the skin is completely covered in a film of sweat, evaporation rate is determined by physical factors (air water vapour pressure, air flow and temperature) rather than by physiological mechanisms involved in sweat gland activity. It seems reasonable to speculate that local temperature and air flow will be different for a given region of the body during different forms of exercise. For example during leg exercise it is possible that metabolic heat produced by the active muscles will lead to an increase in the temperature of the skin of the legs, by conduction through the underlying tissues. Such heat flow and the locally enhanced air flow (due to limb movement), leading to skin cooling and an increased rate of removal of water vapour from the skin surface, might be expected to modify sweat evaporation.

In addition a net change in skin temperature could affect sweat production either by a direct effect on the sweat glands or by modifying the rate of sweat secretion initiated by sudomotor stimuli from the CNS (Kerslake, 1972). Unless the skin is covered with sweat such a modification of sweat production would be associated with a modification of sweat evaporation under the same environmental conditions.

Nielsen (1968) has previously reported that the changes in oesophageal temperature and sweat rate are similar during arm and leg exercise. Sweat evaporation rate was derived from changes in body weight which do not give any information about regional variations in sweating. The present study was designed to investigate regional variations in sweat evaporation rate during arm and leg exercise.

\section{METHODS}

Seven men gave written informed consent to take part in the studies which were approved by the Subcommittee on Practical Procedures, Faculty of Medicine, University of Leeds. The age, weight and height of the subjects (mean \pm SD) were $26 \pm 6$ years (range, $21-34$ years), $73.41 \pm 6.43 \mathrm{~kg}$ (range, 65.23-83.64 kg), and $1.78 \pm 0.04 \mathrm{~m}$ (range, $1.72-1.84 \mathrm{~m}$ ). All took part in physical activity regularly. The maximum oxygen uptake of each subject was predicted on two occasions from measurements of heart rate and oxygen consumption made during steady-state submaximal work (147 watts) using a mechanically-braked (Monark) cycle ergometer (Ástrand and Ryhming, 1954). The predicted maximum oxygen uptake of the group, using the average of the two results for each subject, was $3.46 \pm 0.49 \mathrm{I}_{\mathrm{min}} \mathrm{m}^{-1}$ (range 2.83-4.07 $\left.\mathrm{I} \cdot \mathrm{min}^{-1}\right)$. Each subject took part in two further experiments on different days, no more than two weeks apart, the first involving leg and the second arm cycling.

Each experiment comprised a 35-minute control period, a 15-minute period of leg or arm cycling at approximately $32 \% \mathrm{VO}$ max, and a 15-minute recovery period. In preliminary studies this was the highest relative exercise intensity that could be tolerated for the 15 minutes of arm cycling. The leg exercise was carried out using a mechanically-braked cycle ergometer (Monark) and the arm exercise using a mechanically-braked arm cycle ergometer (Monark Rehabilitation Trainer). In both cases a frequency of $\mathbf{5 0}$ revolutions per minute was used, with a power output of 50-75 W for legs and 25-37 $\mathrm{W}$ for arms. The work load was adjusted during the first 2 minutes of arm exercise to give approximately the same heart rate as the leg exercise. The first measurement of oxygen consumption during exercise was made after 5 minutes. The subjects completed 15 minutes of exercise if oxygen consumption was between 30 and $35 \%$ of the predicted maximum.

The leg experiments took place in a dry bulb temperature of $25.4 \pm 0.4^{\circ} \mathrm{C}$, relative humidity $54.9 \pm 4.0 \%$ and those for the arm $25.3 \pm 0.4^{\circ} \mathrm{C}$, and $51.5 \pm 4.7 \%$.

Aural temperature, skin temperatures (upper arm, upper chest, upper back and thigh), sweat evaporation rate (from the upper arm, upper chest, upper back and thigh), heart rate and respiratory gas exchange were measured at 5 minute intervals during the last 15 mins of the control period, the $15 \mathrm{~min}$ exercise period and the $15 \mathrm{~min}$ recovery period. Sweat evaporation rate was measured at times 0-2, 5-7, 10-12 minutes and so on while respiratory gas exchange, heart rate and body temperatures were measured simultaneously at times 3-5, 8-10, 13-15 minutes and so on. Time 0 was set at 20 minutes after the start of the 35 minute control period.

Heart rate was monitored using precordial ECG electrodes. Oxygen consumption was measured using an automated system (Jaeger Ergooxyscreen). The equipment comprises a paramagnetic oxygen analyser, infrared carbon dioxide analyser and a fleish type pneumotachometer, heated to approximately $40^{\circ} \mathrm{C}$, for the measurement of gas volume. Oxygen consumption was calculated from measurements of expired oxygen, carbon dioxide and gas volume at 30 -second intervals using a microprocessor incorporated in the system. Heat production was derived from measurements of the fraction of expired oxygen and expired gas volume using the formula of Weir (1949). All the measurements were corrected to standard temperature and pressure and dry.

Aural temperature was measured using a thermistor probe (Edale $\mathrm{C}$ ) embedded in a plastic earmould and insulated with foam padding. The thermistor did not come into contact with the tympanic membrane. Skin temperature was measured at 4 sites, anterior medial surface of the mid upper right arm, upper right chest overlying the second intercostal space in the anterior axillary line, upper right back in a similar position to the chest on the posterior axillary line and the anterior surface of the middle of the right thigh. These measurements were made using thermistors (Edale C) which were taped to the skin surface. Mean skin temperature was calculated as $(0.25$ chest +0.25 back +0.3 thigh +0.2 arm). The calculated mean skin temperature using this weighting system compares with the weightings of Ramanathan (1964), Burton (1934) and Newburgh and Spealman (1943) during the same experimental protocol (Ayling, unpublished observations).

Sweat evaporation rate was measured close to the same sites as skin temperature using the ventilated method similar to that described by Lamke (1970) and modified in this laboratory (Ayling, 1985). The system comprises a dewpoint hygrometer (Series 2000, Mitchell), a measuring cup, and a 
diaphragm pump (Charles Austen) to pump air through two dewpoint sensors. One sensor is used to monitor the humidity of room air and one is used to monitor the humidity of the air immediately above the skin surface. The method for measuring sweat evaporation rate by this method has been described previously (Ayling, 1985).

Mean sweat evaporation rate was calculated as the mean of the four sites measured. The mean of the back, chest, forearm and thigh has previously been shown to compare well with measurements of total weight loss during body heating (Lamke and Wedin, 1971).

All results are expressed as the mean \pm 1 standard deviation. Statistical analyses were performed using the paired t-test and the single factor analysis of variance.

\section{RESULTS}

The results are summarised in Tables I and II.

TABLE I

Thermoregulatory responses to leg cycling $n=7$; values are mean \pm 1 SD

\begin{tabular}{lrrr}
\hline Variable & Base Line & $\begin{array}{c}\text { End of } \\
\text { Exercise }\end{array}$ & \multicolumn{1}{c}{$\begin{array}{c}\text { End of } \\
\text { Recovery }\end{array}$} \\
\hline Heart Rate bt.min $^{-1}$ & $69.00 \pm 11.00$ & $112.00 \pm 6.00$ & $70.00 \pm 6.00$ \\
Heat Production W & $80.00 \pm 35.00$ & $508.30 \pm 98.30$ & $111.70 \pm 53.30$ \\
Core Temperature ${ }^{\circ} \mathrm{C}$ & $36.72 \pm 0.28$ & $37.00 \pm 0.30$ & $36.97 \pm 0.28$ \\
Skin Temperature ${ }^{\circ} \mathrm{C}$ & & & \\
Arm & $33.90 \pm 0.60$ & $34.20 \pm 0.80$ & $33.60 \pm 1.40$ \\
Chest & $34.90 \pm 0.60$ & $34.80 \pm 0.70$ & $34.80 \pm 0.60$ \\
Back & $34.40 \pm 0.40$ & $34.10 \pm 0.80$ & $33.40 \pm 1.30$ \\
Thigh & $32.80 \pm 0.50$ & $32.80 \pm 0.90$ & $33.50 \pm 0.80$ \\
Mean & $33.90 \pm 0.40$ & $33.90 \pm 0.50$ & $33.70 \pm 0.70$ \\
Sweat Evaporation & & & \\
mg.cm & & & \\
Arm & & & \\
Chest & $0.70 \pm 0.80$ & $12.80 \pm 17.90$ & $5.10 \pm 7.00$ \\
Back & $0.90 \pm 1.30$ & $19.60 \pm 20.20$ & $4.90 \pm 6.40$ \\
Thigh & $1.30 \pm 1.20$ & $24.80 \pm 20.30$ & $10.10 \pm 16.00$ \\
Mean & $1.60 \pm 1.80$ & $14.70 \pm 12.60$ & $5.10 \pm 6.20$ \\
\hline
\end{tabular}

\section{TABLE ॥}

Thermoregulatory responses to arm cycling. $n=7$; values are mean \pm 1 SD

\begin{tabular}{lcccc}
\hline Variable & Base Line & $\begin{array}{c}\text { End of } \\
\text { Exercise }\end{array}$ & $\begin{array}{c}\text { End of } \\
\text { Recovery }\end{array}$ \\
\hline Heart Rate bt.min ${ }^{-1}$ & $53.00 \pm 4.00$ & $107.00 \pm 12.00$ & $58.00 \pm 5.00$ \\
Heat Production W & $70.00 \pm 35.00$ & $493.30 \pm 193.30$ & $95.00 \pm 57.00$ \\
Core Temperature ${ }^{\circ} \mathrm{C}$ & $36.47 \pm 0.25$ & $36.79 \pm 0.20$ & $36.71 \pm 0.25$ \\
Skin Temperature ${ }^{\circ} \mathrm{C}$ & & & & \\
Arm & $33.10 \pm 1.00$ & $33.70 \pm 1.00$ & $33.60 \pm 1.00$ \\
Chest & $34.30 \pm 0.70$ & $35.00 \pm 0.40$ & $34.50 \pm 0.90$ \\
Back & $34.10 \pm 0.40$ & $34.40 \pm 0.50$ & $33.80 \pm 0.80$ \\
Thigh & $33.00 \pm 1.00$ & $33.80 \pm 1.40$ & $33.00 \pm 1.00$ \\
Mean & $33.60 \pm 0.70$ & $34.20 \pm$ & 0.60 & $33.70 \pm 0.60$ \\
Sweat Evaporation & & & & \\
mg.cm ${ }^{-2}$ hr $^{-1}$ & & & & \\
Arm & $0.70 \pm 1.10$ & $12.20 \pm 7.67$ & $2.90 \pm 3.80$ \\
Chest & $0.40 \pm 0.70$ & $22.20 \pm 14.42$ & $2.50 \pm 3.80$ \\
Back & $2.00 \pm 0.60$ & $27.60 \pm 14.71$ & $2.30 \pm 3.60$ \\
Thigh & $0.30 \pm 0.80$ & $19.20 \pm 13.30$ & $2.50 \pm 3.00$ \\
Mean & $0.40 \pm 0.80$ & $20.30 \pm 11.84$ & $2.60 \pm 3.50$ \\
\hline
\end{tabular}

\section{Heart rate}

Heart rate increased similarly during leg and arm cycling. The difference in resting heart rate between the two studies is significant $(p<0.01)$.

\section{Heat production}

Heat production increased similarly in both studies. In 4 subjects heat production was greater during leg exercise and in 3 subjects it was greater during arm exercise.

\section{Aural temperature}

Aural temperature increased similarly in both experiments. There were no systematic differences in aural temperature during leg and arm cycling with 2 subjects showing a greater rise in aural temperature during leg exercise and 5 during arm exercise. By the end of the 15 minute recovery period aural temperature had started to decrease in both studies.

\section{Skin temperature}

Mean skin temperature decreased during leg cycling from $33.9 \pm 0.4$ to a nadir of $33.6 \pm 0.6^{\circ} \mathrm{C} 8 \mathrm{mins}$ after exercise. In contrast, during arm cycling mean skin temperature increased from $33.6 \pm 0.7$ to $34.2 \pm 0.6$ at the end of the 15 minute exercise period before decreasing to $33.6 \pm 0.6^{\circ} \mathrm{C}$ 8 mins after exercise.

The changes in skin temperature were highly varied between subjects although there was a tendency for the skin temperature to rise at each site during the exercise period and then to decrease at the end of exercise and during recovery. Skin temperature increased slightly more during arm cycling than during leg cycling but this was not associated with any differences in regional sweat evaporation rates.

\section{Sweat evaporation}

There were marked intersubject variations in sweat evaporation rate. Mean sweat evaporation rates were significantly different between individuals during the period of exercise in both studies $(p<0.01)$. The range of mean peak rates was $3.5-54.5 \mathrm{mg} . \mathrm{cm}^{-2} \mathrm{hr}^{-1}$ during leg exercise and 7.1-46.5 mg.cm ${ }^{-2} \mathrm{hr}^{-1}$ during arm exercise. The same two subjects were at each end of the range in both experiments. The subject with the lowest sweating response also showed the greatest rise in core temperature on both occasions $\left(+0.54\right.$ during leg cycling and $+0.86^{\circ} \mathrm{C}$ during arm cycling).

The numerical differences in sweat evaporation rate between the different sites are not statistically significant at any time in either study. There were no systematic differences in mean sweat evaporation rate between the two studies with 3 subjects showing an enhanced overall response during leg exercise and 4 during arm exercise. The mean data suggest an earlier sweating response during arm work although this was not a consistent finding for all subjects.

\section{DISCUSSION}

The subjects who took part in this study were all active but only 3 of the 7 had taken part in laboratory studies of this nature before. This may explain the difference in resting heart rate between the 2 studies. The higher heart rate at the start of the leg cycling study may be due to greater apprehension by some subjects during the leg cycling study which was always carried out first.

Arm cycling is expected to produce higher heart rates at any given oxygen consumption compared with leg cycling with a greater discrepancy being observed as the work load increases (Asmussen and Hemmingsen, 1958). The similarity between heart rates and oxygen consumption seen in the present studies is consistent with the relatively low exercise intensity used, and suggests that the physiological strain was similar in the two situations.

It was interesting that in preliminary studies subjects reported that they could not tolerate arm exercise at about

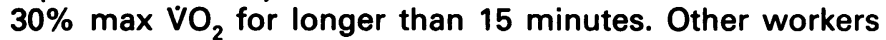
have found that subjects could tolerate this intensity of work for one hour (Nielsen, 1968; Pimental et al, 1984). This 
discrepancy might be due to differences in ergometer design (these authors modified 2 leg cycle ergometers for arm cycling) which might lead to differences in muscular involvement.

No significant differences in regional sweat evaporation rates were observed in the present studies. Thus, although local axon reflexes may be involved in the sweating response to exercise, any influence of such reflexes on sweating from locally active areas of the body is not evident in the present study.

Complete evaporation of sweat was never observed in either study. However, the subjects appeared to sweat more profusely during arm cycling. Despite apparent differences in sweat production during the two forms of exercise the amount of sweat evaporated was not significantly different. This is presumably explained by environmental factors (air water vapour pressure, air flow and temperature) which were similar in both studies.

Four of the 7 subjects appeared to be covered in sweat, with sweat dripping off the skin in these cases. Under such conditions sweat evaporation rate would be maximal. Although physiological factors such as heat acclimatisation and physical training modify sweat production, any differences in sweat production would not be expected to lead to differences in sweat evaporation between subjects in the same environmental conditions, with the same skin temperature and whose sweat production exceeds sweat evaporation. Since the variation in sweat evaporation rate between subjects exceeded the variation that could be attributed to error in the measurement this anomaly is most easily explained by an inaccuracy in the subjective assessment of sweating intensity.

In summary, in the present study a short period of arm or leg cycling was associated with similar regional sweat evaporation rates for individual subjects. The present study has not eliminated the possibility that there are regional differences in sweat evaporation rate during longer term exercise which is associated with steady state sweating.

\section{References}

Asmussen, E. and Hemmingsen, I., 1958 "Determination of maximal working capacity at different ages in work with the legs or with the arms". Scand.J.Clin.Lab.Invest. 10: 67-71.

Astrand, P. O. and Ryhming, I., 1954 "A nomogram for calculation of aerobic capacity (physical fitness) from pulse rate during submaximal work". J.Appl.Physiol. 7: 218-221.

Ayling, J. H., 1985 "Use of dew-point sensors for intermittent measurements of sweat production and evaporation rates". J.Sports Med.Phys.Fitness. In press.

Beaumont, Van W. and Bullard, R. W., 1963 "Sweating: Its rapid response to muscular work". Science 141: 643-646.

Burton, A. C., 1934 "The applications of the theory of heat flow to the study of energy metabolism". J.Nutr. 7: 497-533.

Davies, C. T. M., 1979 "The effects of different levels of heat production induced by diathermy and eccentric work on thermoregulation during exercise at a given skin temperature". Eur.J.Appl.Physiol. 40: 171-180.

Kerslake, D. McK., 1972. The Stress of Hot Environments. p. 142. University Press, Cambridge.

Lamke, L. O., 1970 "An instrument for estimation of water evaporation from small skin surfaces". Scand.J.Plast.Recon.Surg.(Stockholm) 4: 1-7.

Lamke, L. O. and Wedin, B., 1971 "Water evaporation from normal skin under different environmental conditions". Acta Dermatovener (Stockholm) 51 : 111-119.

Newburgh, L.H. and Spealman, C. R., 1943 "Acclimatisation to cold". CAM Report No. 241. Cited by Teichner, W. H. (1958). J.Appl.Physiol. 12: $169-176$

Nielsen, B. G., 1968 "Thermoregulatory responses to arm work, leg work and intermittent leg work". Acta Physiol.Scand. 72: 25-32.

Nielsen, B., 1969 "Thermoregulation in rest and exercise". Acta Physiol.Scand.(Suppl.) 323: 10-73.

Pimental, N. A.,Suaka, M. N., Billings, D. S. andTrad, L.A., 1984 “Physiological responses to prolonged upper-body exercise". Med.Sci.Sports Exerc. 16: 360-365.

Ramanathan, N. L., 1964 "A new weighting system for mean surface temperature of the human body". J.Appl.Physiol. 19: 531-533.

Weir, V. de J. B., 1949 "New methods for calculating metabolic rate with special reference to protein metabolism". J.Physiol. 109: 1-9. 